hep-th/0011122

UT-KOMABA/00-14

November 2000

\title{
Supercurrents in Matrix theory and the generalized AdS/CFT correspondence
}

\author{
Yasuhiro Sekino 目 \\ Institute of Physics \\ University of Tokyo, Komaba, Tokyo 153
}

\begin{abstract}
We investigate Matrix theory in the large- $N$ limit following the conjectured correspondence between Matrix theory and supergravity on the near-horizon limit of the D0-brane background. We analyze the complete fermionic spectrum of supergravity and obtain twopoint functions of the supercurrents in Matrix theory. By examining the large- $N$ scaling properties of the correlators, we analyze the behavior of the supercurrents under the boost in the 11-th direction and discuss the consistency of the 11-dimensional interpretation of the supersymmetry of Matrix theory.
\end{abstract}

*e-mail address: sekino@hep1.c.u-tokyo.ac.jp 


\section{Introduction}

According to the Matrix-theory conjecture of Banks, Fischler, Shenker and Susskind [1], M-theory in the infinite momentum frame is defined by the large- $N$ limit of the onedimensional $\mathrm{U}(N)$ super Yang-Mills theory which describe the collection of $N$ D0-branes. Since the D0-brane is believed to be the Kaluza-Klein particle corresponding to the compactification of 11-th direction and has positive longitudinal momentum, it is considered as a natural candidate for the elementary degrees of freedom in the infinite momentum frame. Also, extended objects of M-theory such as membranes and 5-branes can be described as classical configurations of matrices in SYM theory. However, what is highly non-trivial is that the SYM theory which is the description of D-branes in the low-energy, short-distance and the weak-coupling limit is proposed as the exact non-perturbative definition of M-theory. Taking the large- $N$ limit must be an important ingredient. Subsequently, it was proposed that the finite- $N$ version of Matrix theory can be interpreted as the compactification of M-theory on a lightlike circle [2]. The compactification in the lightlike direction is equivalent to the compactification in the spacelike direction with small compactification radius and small transverse length scale [3, 4. Since M-theory compactified on the small spatial circle is the weakly-coupled type IIA string theory, it provides the explanation for the appearance of SYM theory for the D0-branes. Various quantitative checks for the finite- $N$ interpretation has been performed, including the consistency with the supergravity interactions up to two-loop perturbations in Matrix theory [5, 6, 7].

As Matrix theory is intrinsically formulated in 10-dimensional language, the crucial issue is how it realizes the 11-dimensional Lorentz invariance. In Matrix theory, the longitudinal momentum is given by $P_{-}=N / R$ where $R=g_{s} \ell_{s}$ and the boost in the 11-th direction rescales $N$ with fixed $g_{s}$, according to the proposal of Banks et al. Thus, for the boost invariance, the theory must have a kind of scale invariance with respect to $N$ in the large $N$ limit. However, analysis of gauge theory in the large $N$ limit (with fixed $g_{s}$ ) is difficult and there was no clue for this 'scale invariance'.

In the previous paper [11], with the motivation for understanding the boost invariance of Matrix theory, we have investigated Matrix theory in the large- $N$ limit using the con- 
jectured correspondence with supergravity. For non-dilatonic branes such as D3-branes, exact equivalence between super Yang-Mills theory describing the branes and the superstring theory on the near-horizon limit of the classical solution is proposed [ $\mathbb{B}$. The same kind of correspondence is conjectured for dilatonic branes such as D0-branes [9]. The conjecture was made more precise by Jevicki and Yoneya [10] who pointed out that Matrix theory and the near-horizon limit of the D0-brane solution have the same symmetry called the generalized conformal symmetry. In [11], following this 'generalized AdS/CFT correspondence' and assuming the relation between the supergravity action evaluated on the near-horizon limit of D0-brane solution and the generating functional for Matrix-theory correlators, following the general method proposed by Gubser, Klebanov and Polyakov [12] and by Witten [13] for non-dilatonic branes, we have predicted the 2-point functions of bosonic operators of Matrix theory. We have examined the $N$ dependence of the correlators and analyzed the behavior of Matrix-theory operators under the boost in the 11-th direction. We have found that the scaling of $N$ can be interpreted as a spacetime symmetry, but there was an unexpected anomalous behavior for interpreting it as the Lorentz boost. However, this anomalous behavior does not necessarily mean the violation of 11-dimensional Lorentz invariance in Matrix theory. We have to point out the possibility that the generalized AdS/CFT correspondence does not give the exact information for Matrix theory, which may be due to the fact that we have to restrict ourselves to the near-horizon region of D0-branes in the supergravity calculation in this formulation. We shall review these discussions in section 4 of this paper for completeness.

In this paper, we study the Matrix-theory supercurrents. The boost properties of the supercurrents are particularly important, for they form the basis for the following 11-dimensional interpretation of the supersymmetry of Matrix theory. The SUSY in 11 dimensions has 32 real supercharges. In the lightcone frame where $\mathrm{SO}(9)$ of $\mathrm{SO}(10,1) \supset$ $\mathrm{SO}(1,1) \times \mathrm{SO}(9)$ is manifest, supercharges are divided into two classes which are called the kinematical SUSY and the dynamical SUSY, according to the $\mathrm{SO}(1,1)$ weight (longitudinal boost weight). The explanation for the 11-dimensional SUSY in Matrix theory is as follows. Matrix theory has two kinds of fermionic symmetries: one is a constant shift of fermionic field only, and the other is the usual SUSY transformation of SYM theory. The former is interpreted as the kinematical SUSY and the latter is interpreted as the 
dynamical SUSY in 11 dimensions. Indeed, the Dirac-bracket algebra of the supercharges calculated in Matrix theory reproduces the 11-dimensional lightcone SUSY algebra under this identification [14]. In this paper, we try to give further evidence for the 11-dimensional SUSY of Matrix theory by checking whether the supercharges (supercurrents) have the correct $\mathrm{SO}(1,1)$ weight in the sense of the large- $N$ scaling behavior. We continue the analysis of [11] and compute the two-point functions of the supercurrents of Matrix theory following the generalized AdS/CFT correspondence. For that purpose, we work out the complete spectrum of the fermionic degrees of freedom of 10-dimensional type IIA supergravity on the near-horizon limit of D0-brane background. Analysis of the spectrum is performed on the equivalent 11-dimensional background. We analyze the $N$-dependence of the correlators which are obtained form the generalized AdS/CFT correspondence and discuss the behavior of the supercharges under the boost in the 11-th direction.

This paper is organized as follows. In section 2, we review the 10- and 11-dimensional descriptions of D0-brane solution. In section 3, the fermionic spectrum of supergravity is analyzed. In section 4, we calculate Matrix-theory two-point functions following the generalized AdS/CFT correspondence. In section 5, we discuss the 11-dimensional Lorentz invariance and supersymmetry of Matrix theory by analyzing the scaling behavior of the correlators with respect to $N$. We conclude in section 6 .

\section{Ten- and eleven- dimensional descriptions of the solution}

\subsection{The D0-brane solution}

D0-brane solution in the string frame in 10 dimensions is given by

$$
\begin{aligned}
& d s_{10}^{2}=-\mathrm{e}^{2 \tilde{\phi} / 3} d t^{2}+\mathrm{e}^{2 \tilde{\phi} / 3} d x_{m}^{2}, \\
& \mathrm{e}^{\phi}=g_{s} \mathrm{e}^{\tilde{\phi}}, \quad A_{0}=-\frac{1}{g_{s}}\left(\frac{1}{1+h}-1\right) \\
& \mathrm{e}^{\tilde{\phi}}=(1+h)^{3 / 4},
\end{aligned}
$$

where

$$
h=\frac{q}{r^{7}}, \quad q=60 \pi^{3}\left(\alpha^{\prime}\right)^{7 / 2} g_{s} N .
$$

and $m(=1, \ldots, 9)$ denotes the indices of the Cartesian coordinates in the transverse directions throughout this paper. String coupling $g_{s}$ is defined by $\mathrm{e}^{\phi}$ at infinity and the 
part of the dilaton which vanish at infinity is denoted by $\tilde{\phi}$.

The D0-brane solution can be considered as the dimensional reduction of the solution in 11 dimensions. 10-dimensional description and 11-dimensional description are equivalent if we note a few points remarked in the next subsection. We use the 11-dimensional description for the analysis of the spectrum in the next section, and we convert to the 10-dimensional language when studying the generalized AdS/CFT correspondence which is formulated in 10 dimensions. The metric in 11 dimensions and the metric, dilaton and one-form field in 10 dimensions are related by

$$
d s_{11}^{2}=\mathrm{e}^{-2 \phi / 3} d s_{10}^{2}+\mathrm{e}^{4 \phi / 3}\left(d x^{11}-A_{\mu} d x^{\mu}\right)^{2}
$$

The metric which yields the D0-brane solution upon dimensional reduction on $x^{11}$ is given by䗆

$$
d s^{2}=d x^{+} d x^{-}+h d x^{-} d x^{-}+d x_{m}^{2}
$$

where $x^{ \pm}= \pm t+x^{11}$. We choose the following form of vielbein.

$$
e_{+}^{\hat{0}}=\frac{1}{2 \sqrt{h}}, \quad e^{\hat{11}}=\frac{1}{2 \sqrt{h}}, \quad e_{-}^{\hat{0}}=0, \quad e_{-}^{\hat{11}}=\sqrt{h}
$$

Indices with hat refers to those for the local Lorentz frame throughout this paper. We summarize the connections and the curvatures of this background in Appendix A.

\subsection{Near-horizon limit}

The 11-dimensional geometry given by (2.3) corresponds to the full D0-brane solution (without taking near-horizon limit). The near-horizon limit $\left(r \ll q^{1 / 7}\right)$ of the D0-brane solution in 10 dimensions is given by substituting $h$ for $1+h$ in (2.1). That metric is formally obtained from the metric

$$
d s^{2}=2 d t d x^{-}+h d x^{-} d x^{-}+d x_{m}^{2}
$$

${ }^{\dagger}$ To derive (2.3) from (2.1) using (2.2), we have rescaled the value of the coordinates

$$
x^{(11) \mu}=g_{s}^{-1 / 3} x^{(10) \mu}, \quad(\mu=0, \ldots 9), \quad x^{(11) 11}=g_{s}^{2 / 3} x^{(10) 11} .
$$

where $x^{(11) \mu}, x^{(11) 11}$ are the coordinates used in (2.3). With the rescaling of $x^{\mu}$, asymptotic flat 11D metric corresponds to asymptotic flat 10D metric. The rescaling of the 11-th coordinate is such that the period for the compactification is $R=g_{s}^{3 / 2} \ell_{P}=g_{s} \ell_{s}\left(\right.$ not $\left.=\ell_{P}\right)$ for $x^{(11)}$. 
by dimensional reduction on the $x^{11}$ direction. (2.5) means that we can analyze the near-horizon limit of the 10-dimensional solution in the 11-dimensional language, by reinterpreting the coordinate as follows.

$$
2 t^{(n . h .)}=x^{(f u l l)+}, \quad x^{(n . h .)-}=x^{(f u l l)-}
$$

where $x^{(f u l l)}$ is the coordinate which was used in the last subsection. We use the coordinate $x^{(f u l l)}$ in the calculation and make this reinterpretation at the end.

There is one thing which needs care. We want to analyze the spectrum of the fluctuations which does not depend on the 11-th coordinate, for it is directly related to the 10dimensional interpretation. Here, 11-th coordinate mean $x^{(n . h .) 11}$ when we want to study the near-horizon limit. As the fluctuations are independent of $x^{(n . h .) 11}=\frac{1}{2} x^{(f u l l)+}+x^{(f u l l)-}$ (but depends on $t^{(n . h .)}=\frac{1}{2} x^{(f u l l)+}$ and other transverse coordinates), in the intermediate stage where we are calculating using the '( $f u l l)$ 'coordinate, we must take the fluctuations

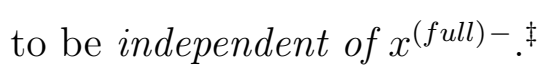

\section{Fermionic spectrum on the near-horizon D0-brane background}

\subsection{Equations of motion and SUSY invariance}

We shall analyze the spectrum of the fermionic fluctuations on the background (2.3). Fermionic fields of the 11-dimensional supergravity are the Rarita-Schwinger fields $\Psi_{M}$ which are Majorana spinors having vector indices. (We use $M=0,1, \ldots, 9,11$ for the indices of the 11-dimensional coordinate throughout this paper.) Linearized equation of motion for the RS field is

$$
\Gamma^{M N P} D_{N} \Psi_{P}=0
$$

where $D_{M}$ is the covariant derivative containing the torsionless spin connection at the linearized level. Equations of motion for each component is given as follows. We use the spherical coordinates on the transverse $S^{8}$, which is denoted by $x^{i}(i=1, \ldots, 8)$. In the following equations, we use the 'covariant derivative on the unit sphere' $\tilde{D}_{i}$ which is made from the connections on $S^{8}$. Covariant derivative $D_{i}$ on the background (2.3) is not equal

\footnotetext{
17.

${ }^{\ddagger}$ Compactification of the supergravity solution $(2.3)$ on the lightlike circle is also discussed in 15,16 ,
} 
to $\tilde{D}_{i}$, for $D_{i}$ has extra contributions from $\Gamma^{r}{ }_{i j}, \Gamma_{r j}^{i}$ and $\omega_{i r \hat{\jmath}}$. Note that the background (2.3) is not a direct product of a sphere and something. (See Appendix A.) The $(M=+)$ component of (3.1) is

$$
\begin{gathered}
\Gamma^{+-} \Gamma^{r}\left(D_{-} \Psi_{r}-D_{r} \Psi_{-}\right)-\Gamma^{+-} \Gamma^{i} \tilde{D}_{i} \Psi_{-}-\frac{4}{r} \Gamma_{r} \Gamma^{+-} \Psi_{-}-\Gamma^{+} \Gamma^{r} \Gamma^{i} \tilde{D}_{i} \Psi_{r}-\frac{4}{r} \Gamma^{+} \Psi_{r} \\
-\Gamma^{+} \tilde{D}^{i} \Psi_{i}+\Gamma^{+-} D_{-} \Gamma^{i} \Psi_{i}+\Gamma^{+} \Gamma^{r} D_{r} \Gamma^{i} \Psi_{i}+\Gamma^{+} \Gamma^{j} \tilde{D}_{j} \Gamma^{i} \Psi_{i}+\frac{9}{2 r} \Gamma^{+} \Gamma_{r} \Gamma^{i} \Psi_{i}=0
\end{gathered}
$$

The $(M=-)$ component is

$$
\begin{array}{r}
\Gamma^{-+} \Gamma^{r}\left(D_{+} \Psi_{r}-D_{r} \Psi_{+}\right)-\Gamma^{-+} \Gamma^{i} \tilde{D}_{i} \Psi_{+}-\frac{4}{r} \Gamma_{r} \Gamma^{-+} \Psi_{+}-\Gamma^{-} \Gamma^{r} \Gamma^{i} \tilde{D}_{i} \Psi_{r}-\frac{4}{r} \Gamma^{-} \Psi_{r} \\
-\Gamma^{-} \tilde{D}^{i} \Psi_{i}+\Gamma^{-+} D_{+} \Gamma^{i} \Psi_{i}+\Gamma^{-} \Gamma^{r} D_{r} \Gamma^{i} \Psi_{i}+\Gamma^{-} \Gamma^{j} \tilde{D}_{j} \Gamma^{i} \Psi_{i}+\frac{9}{2 r} \Gamma^{-} \Gamma_{r} \Gamma^{i} \Psi_{i}=0
\end{array}
$$

The $(M=r)$ component is

$$
\begin{array}{r}
\Gamma^{+-}\left(D_{+} \Psi_{-}-D_{-} \Psi_{+}\right)-\Gamma^{+} \Gamma^{i} \tilde{D}_{i} \Psi_{+}+\frac{4}{r} \Gamma_{r} \Gamma^{+} \Psi_{+}-\Gamma^{-} \Gamma^{i} \tilde{D}_{i} \Psi_{-}+\frac{4}{r} \Gamma_{r} \Gamma^{-} \Psi_{-} \\
-\tilde{D}^{i} \Psi_{i}+\Gamma^{+} D_{+} \Gamma^{i} \Psi_{i}+\Gamma^{-} D_{-} \Gamma^{i} \Psi_{i}+\Gamma^{j} \tilde{D}_{j} \Gamma^{i} \Psi_{i}+\frac{7}{2 r} \Gamma_{r} \Gamma^{i} \Psi_{i}=0
\end{array}
$$

The $(M=i)$ component contracted with $\Gamma^{i}$ (after subtraction of a certain combination of $(3.2),(3.3)$ and $(3.4))$ is

$$
\begin{array}{r}
-\Gamma^{+} \Gamma^{i} \tilde{D}_{i} \Psi_{+}+\frac{4}{r} \Gamma_{r} \Gamma^{+} \Psi_{+}-\Gamma^{-} \Gamma^{i} \tilde{D}_{i} \Psi_{-}+\frac{4}{r} \Gamma_{r} \Gamma^{-} \Psi_{-}-\Gamma^{r} \Gamma^{i} \tilde{D}_{i} \Psi_{r}-\frac{4}{r} \Psi_{r}-2 \tilde{D}^{i} \Psi_{i} \\
+\Gamma^{+} D_{+} \Gamma^{i} \Psi_{i}+\Gamma^{-} D_{-} \Gamma^{i} \Psi_{i}+\Gamma^{r} D_{r} \Gamma^{i} \Psi_{i}+2 \Gamma^{j} \tilde{D}_{j} \Gamma^{i} \Psi_{i}+\frac{8}{r} \Gamma_{r} \Gamma^{i} \Psi_{i}=0
\end{array}
$$

The ' $\Gamma^{i}$-transverse' part of $(M=i)$ component (which vanishes when contracted with $\Gamma^{i}$ ) is

$$
-\Gamma^{\alpha} \tilde{D}_{(i)} \Psi_{\alpha}+\Gamma^{\alpha} D_{\alpha} \Psi_{(i)}-\Gamma^{r} \tilde{D}_{(i)} \Psi_{r}+\Gamma^{r} D_{r} \Psi_{(i)}+\Gamma^{j} \tilde{D}_{j} \Psi_{(i)}+\frac{4}{r} \Gamma_{r} \Psi_{(i)}=0
$$

where the subscript $(i)$ means that appropriate term proportional to $\Gamma^{i}$ is substracted to make the expression $\Gamma^{i}$-transverse.

The equations of motion are invariant under the local supersymmetry under which the linearized fluctuations transform as $\delta \Psi_{M}=D_{M} \epsilon$. For general modes, we fix this gauge invariance by imposing the condition

$$
\Gamma^{i} \Psi_{i}=0
$$


For the modes with lowest angular momentum, the above condition cannot be imposed since some of the components of the fluctuations are invariant under the SUSY transformation, as we shall see in section 3.4. That case will be treated separately.

We take the following explicit representation for the $\Gamma$ matrices in 11 dimensions which have $32 \times 32$ components.

$$
\Gamma^{\hat{0}}=i \sigma^{1} \otimes \gamma^{9}, \Gamma^{\hat{r}}=\sigma^{2} \otimes \gamma^{9}, \Gamma^{\hat{11}}=\sigma^{3} \otimes \gamma^{9}, \Gamma^{\hat{\imath}}=1 \otimes \gamma^{\hat{\imath}}
$$

where $\gamma^{\hat{\imath}}$ are Gamma matrices in 8 dimensions $(16 \times 16$ matrices $)$ which satisfy $\left\{\gamma^{\hat{\imath}}, \gamma^{\hat{\jmath}}\right\}=$ $2 \delta^{\hat{\imath} \hat{\jmath}}$ and $\gamma^{9}=\gamma^{1} \cdots \gamma^{8}$.

\subsection{Expansions into spinor and vector-spinor spherical harmonics}

Since the background has $\mathrm{SO}(9)$ symmetry in the transverse space, it is appropriate to expand each field into spherical harmonics which transform irreducibly under $\mathrm{SO}(9)$.

We decompose the spinor fields on $S^{8}\left(\Psi_{+}, \Psi_{-}\right.$and $\left.\Psi_{r}\right)$ into spinor spherical harmonics $\left(\Xi^{k,+}, \Xi^{k,-}\right)$ on $S^{8}$ which are 16-component Majorana spinors. The coefficients of the expansion $\left(\hat{\Psi}_{+}, \hat{\Psi}_{-}\right.$and $\left.\hat{\Psi}_{r}\right)$ are 2-component spinors.

$$
\begin{aligned}
& \Psi_{+}=\sum_{+,-} \sum_{k=0}^{\infty} \hat{\Psi}_{+}^{k, \pm}\left(\gamma^{9}\right)^{-1 / 2} \Xi^{k, \pm}, \quad \Psi_{-}=\sum_{+,-k=0} \sum_{-}^{\infty} \hat{\Psi}_{-}^{k, \pm}\left(\gamma^{9}\right)^{-1 / 2} \Xi^{k, \pm} \\
& \Psi_{r}=\sum_{+,-} \sum_{k=0}^{\infty} \hat{\Psi}_{r}^{k, \pm}\left(\gamma^{9}\right)^{-1 / 2} \Xi^{k, \pm}
\end{aligned}
$$

Spinor spherical harmonics are the eigenfunctions of the Dirac operator on the sphere

$$
\gamma^{i} \tilde{D}_{i} \Xi^{k, \pm}=\mp i(k+4) \Xi^{k, \pm} . \quad(k=0,1, \ldots)
$$

As we can see from (3.10), $\Xi^{k,+}$ and $\Xi^{k,-}$ transform to each other when multiplied by $\gamma^{9}$. We have to take a linear combination of $\Xi^{k,+}$ and $\Xi^{k,-}$ in the expansion (3.9) for diagonalizing the equations of motion. (We have denoted the combination by $\left(\gamma^{9}\right)^{-1 / 2}$ following 18]. $\left(\gamma^{9}\right)^{-1 / 2}$ has the property $\gamma^{i}\left(\gamma^{9}\right)^{-1 / 2}=-i\left(\gamma^{9}\right)^{1 / 2} \gamma^{i}$.) The explicit forms of the harmonics $\Xi^{k, \pm}$ are given by

$$
\Xi^{k, \pm}=\left[\left(k+7 \pm i \gamma^{i} \tilde{D}_{i}\right) Y^{k}\right] \eta^{ \pm}
$$


where $Y^{k}$ is the scalar spherical harmonics of order $k$ which satisfy $\tilde{D}^{2} Y^{k}=-k(k+7) Y^{k}$. $\tilde{D}_{i}$ acts only on $Y^{k}$ in (3.11). $\eta^{ \pm}$are the Killing spinors on $S^{8}$ which satisfy

$$
\tilde{D}_{i} \eta^{ \pm}=\mp \frac{i}{2} \gamma_{i} \eta^{ \pm}
$$

The $k=0$ modes of the spinor spherical harmonics are the Killing spinors themselves.

We expand the vector-spinor on $S^{8}, \Psi_{i}$ which satisfy $\Gamma^{i} \Psi_{i}=0$ as

$$
\Psi_{i}=\sum_{+,-} \sum_{k=1}^{\infty} \hat{\Psi}^{k, \pm}\left(\gamma^{9}\right)^{-1 / 2} \Xi_{i}^{k, \pm}+\sum_{+,-} \sum_{k=1}^{\infty} \hat{\hat{\Psi}}^{k, \pm}\left(\gamma^{9}\right)^{-1 / 2} \tilde{D}_{(i)} \Xi^{k, \pm}
$$

The vector spinor harmonics $\Xi_{i}^{k,+}, \Xi_{i}^{k,-}$ are 16-component Majorana spinors and have the properties

$$
\gamma^{i} \Xi_{i}^{k, \pm}=0, \quad \tilde{D}^{i} \Xi_{i}^{k, \pm}=0
$$

and are the eigenfunctions of the Dirac operator on the sphere

$$
\gamma^{j} \tilde{D}_{j} \Xi_{i}^{k, \pm}=\mp i(k+4) \Xi_{i}^{k, \pm} \quad(k=1,2, \ldots)
$$

$\tilde{D}_{(i)} \Xi^{k, \pm}$ is defined by

$$
\tilde{D}_{(i)} \Xi^{k, \pm}=\tilde{D}_{i} \Xi^{k, \pm} \pm \frac{i}{8}(k+4) \gamma_{i} \Xi^{k, \pm} \quad(k=1,2, \ldots)
$$

and is the ' $\gamma^{i}$-transverse' part of $\tilde{D}_{i} \Xi$. (satisfies $\gamma^{i} \tilde{D}_{(i)} \Xi=0$ ). The $k=0$ mode is an exception. Since the vector-spinor harmonics are defined for $k \geq 1$ and $\tilde{D}_{(i)} \Xi^{k, \pm}$ vanishes for $k=0$ (as we can see from (3.12) and (3.16) ), $\Psi_{i}$ which satisfy $\gamma^{i} \Psi_{i}=0$ does not have the $k=0$ mode. However, we cannot impose the condition $\gamma^{i} \Psi_{i}=0$ in this case (as we will see in section 3.4), and we expand $\Psi_{i}$ for the $k=0$ mode without that condition as

$$
\Psi_{i}=-\sum \hat{\Psi}^{0, \pm} \gamma^{i}\left(\gamma^{9}\right)^{1 / 2} \eta^{ \pm}
$$

Since the spherical harmonics of different kind, or with different $k,+,-$ are orthogonal to each other, we can separately analyze each mode. The superscript $(k,+),(k,-)$ on the spherical harmonics will be often omitted when there is no source of confusion. 


\subsection{Transverse vector-spinor modes}

First, we analyze the mode corresponding to the vector-spinor harmonics $\Xi_{i}^{(k, \pm)}$. We impose the gauge condition $\Gamma^{i} \Psi_{i}=0$ and substitute the harmonic expansions (3.9) and (3.13) into the equations of motion. The vector-spinor modes only appear in (3.6) where the coefficient of $\left(\gamma^{9}\right)^{-1 / 2} \Xi_{i}^{k, \pm}$ is

$$
\left[2 \sqrt{h} i \sigma^{1} \partial_{+}+\frac{1}{\sqrt{h}}\left(-i \sigma^{1}+\sigma^{2}\right) \partial_{-}+\sigma^{3} \partial_{r}+\frac{h^{\prime}}{4 h}+\frac{4}{r} \sigma^{3} \mp \frac{1}{r}(k+4)\right] \hat{\Psi}=0 .
$$

where $h^{\prime}=\partial_{r} h=-7 h / r$.

To analyze the near-horizon limit of the D0-brane solution, we reinterpret $x^{+} \rightarrow 2 t$ and assume the fields to be independent of $x^{-}$, as explained in section 2.2. Then, we change the radial variable to

$$
z=\frac{2}{5} q^{1 / 2} r^{-5 / 2}
$$

and Euclidize the time coordinate $\left(t=-i \tau, \omega_{M}=i \omega_{E}\right)$.

$$
\left[-\sigma^{1} \partial_{\tau}-\sigma^{3} \partial_{z}+\left(\mp \frac{2}{5}(k+4)+\frac{7}{10}\right) \frac{1}{z}\right]\left(z^{-8 / 5} \hat{\Psi}\right)=0
$$

The solution which is regular at $r \rightarrow 0(z \rightarrow \infty)$ is given by

$$
\hat{\Psi}=\int \frac{d \omega}{2 \pi} \mathrm{e}^{-i \omega \tau} z^{\frac{21}{10}}\left\{C^{(1)}(\omega) K_{\frac{2}{5}\left|\frac{1}{2} \mp(k+4)\right|}(|\omega| z)+C^{(2)}(\omega) K_{\frac{2}{5}|3 \mp(k+4)|}(|\omega| z)\right\}
$$

where the superscript (1) denotes the upper component of the two component spinors and (2) denotes the lower component, $\left(\sigma^{3} C^{(1)}=+C^{(1)}\right.$ and $\left.\sigma^{3} C^{(2)}=-C^{(2)}\right)$. To be a solution of $(3.20), C^{(1)}$ and $C^{(2)}$ must satisfy

$$
i \omega \sigma^{1} C^{(1)}=|\omega| C^{(2)}
$$

Note that as is the case for the 1st-order action, half of the components are physical and we can only fix either $\hat{\Psi}^{(1)}$ or $\hat{\Psi}^{(2)}$ by a boundary condition.

\subsection{Spinor modes at the lowest level $(k=0)$}

Next, we analyze the spinor modes on the sphere with lowest angular momentum, $\Xi^{k=0, \pm}$. First of all, we shall discuss the supersymmetry on the D0-brane background. The trans- 
formation $\delta \Psi_{M}=D_{M} \epsilon$ is written in components as

$$
\begin{aligned}
& \delta \Psi_{+}=\partial_{+} \epsilon, \quad \delta \Psi_{-}=\partial_{-} \epsilon+\frac{h^{\prime}}{4 \sqrt{h}}\left(\left(i \sigma^{1}-\sigma^{2}\right) \otimes \mathbb{1}\right) \epsilon, \\
& \delta \Psi_{r}=\partial_{r} \epsilon+\frac{h^{\prime}}{4 h}\left(\sigma^{3} \otimes \mathbb{1}\right) \epsilon, \quad \delta \Psi_{i}=\tilde{D}_{i} \epsilon-\frac{1}{2}\left(\sigma^{3} \otimes \gamma^{9} \gamma_{i}\right) \epsilon
\end{aligned}
$$

When $\epsilon$ is in the $k=0$ mode (proportional to the Killing spinor)

$$
\epsilon=\hat{\epsilon}^{ \pm}\left(\gamma^{9}\right)^{-1 / 2} \eta^{ \pm}
$$

we can see from the property of the Killing spinor (3.12) that $\Psi_{i}$ transforms as

$$
\delta \Psi_{i}=-\frac{1}{2}\left( \pm 1-\sigma^{3}\right) \hat{\epsilon}^{ \pm} \otimes \gamma_{i}\left(\gamma^{9}\right)^{1 / 2} \eta^{ \pm} .
$$

That is, half of the components of $\Psi_{i}$ are invariant under the SUSY and we cannot achieve $\Gamma^{i} \Psi_{i}=0$ for the $k=0$ modes. We shall first analyze the equations for the $k=0$ modes without gauge fixing and later specify a gauge conditions which are convenient.

We have to analyze four equations (3.2) (3.5). (' $\Gamma^{i}$-transverse part (3.6) is identically zero for $k=0$ modes.) Substituting the harmonic expansions (3.9) and (3.17) for $\Psi_{+}$, $\Psi_{-}, \Psi_{r}$ and $\Psi_{i}$, we obtain the following equations for $k=0$ modes without gauge fixing. The upper signs are for $\Xi^{k=0,+}$ mode and the lower signs are for $\Xi^{k=0,-}$. From (3.2),

$$
\begin{aligned}
& \frac{i}{\sqrt{h}} \sigma^{1}\left(\partial_{-} \hat{\Psi}_{r}-\partial_{r} \hat{\Psi}_{-}\right)-\frac{h^{\prime}}{4 h^{3 / 2}} \sigma^{2} \hat{\Psi}_{-}+\frac{4}{\sqrt{h} r}\left(-i \sigma^{1} \pm \sigma^{2}\right) \hat{\Psi}_{-}+\frac{4}{r}\left(-1 \pm \sigma^{3}\right) \hat{\Psi}_{r} \\
& +\frac{h^{\prime}}{4 h}\left(-1+\sigma^{3}\right) \hat{\Psi}_{r}-\frac{8}{\sqrt{h} r} \sigma^{2} \partial_{-} \hat{\Psi}-\frac{8}{r} \sigma^{3} \partial_{r} \hat{\Psi}-\frac{2 h^{\prime}}{h r} \sigma^{3} \hat{\Psi}-\frac{28}{r^{2}}\left( \pm 1+\sigma^{3}\right) \hat{\Psi}=0
\end{aligned}
$$

From (3.3),

$$
\begin{aligned}
& 2\left(\partial_{+} \hat{\Psi}_{r}-\partial_{r} \hat{\Psi}_{+}\right)-\frac{h^{\prime}}{2 h} \sigma^{3} \hat{\Psi}_{+}+\frac{8}{r}\left( \pm \sigma^{3}-1\right) \hat{\Psi}_{+}+(1 \pm 1) \frac{4}{\sqrt{h} r}\left(i \sigma^{1}-\sigma^{2}\right) \hat{\Psi}_{r}-\frac{16}{r} \sigma^{3} \partial_{+} \hat{\Psi} \\
& -\frac{8}{\sqrt{h} r}\left(i \sigma^{1}-\sigma^{2}\right) \partial_{r} \hat{\Psi}+\frac{2 h^{\prime}}{h^{3 / 2} r}\left(i \sigma^{1}-\sigma^{2}\right) \hat{\Psi}+(-1 \pm 1) \frac{28}{\sqrt{h} r^{2}}\left(i \sigma^{1}-\sigma^{2}\right) \hat{\Psi}=0
\end{aligned}
$$

From (3.4),

$$
\begin{gathered}
2 \sigma^{3}\left(\partial_{-} \hat{\Psi}_{+}-\partial_{+} \hat{\Psi}_{-}\right)+\frac{h^{\prime}}{2 h}\left(i \sigma^{1}-\sigma^{2}\right) \hat{\Psi}_{+}+\frac{8 \sqrt{h}}{r}\left( \pm i \sigma^{1}-\sigma^{2}\right) \hat{\Psi}_{+}+(1 \pm 1) \frac{4}{\sqrt{h} r}\left(-i \sigma^{1}+\sigma^{2}\right) \hat{\Psi}_{-} \\
-\frac{16 \sqrt{h}}{r} i \sigma^{1} \partial_{+} \hat{\Psi}-\frac{8}{\sqrt{h} r}\left(-i \sigma^{1}+\sigma^{2}\right) \partial_{-} \hat{\Psi}-\frac{28}{r^{2}}\left( \pm 1+\sigma^{3}\right) \hat{\Psi}=0
\end{gathered}
$$


From (3.5),

$$
\begin{array}{r}
-\frac{\sqrt{h}}{r}\left(\mp i \sigma^{1}+\sigma^{2}\right) \hat{\Psi}_{+}+(1 \pm 1) \frac{1}{2 \sqrt{h} r}\left(-i \sigma^{1}+\sigma^{2}\right) \hat{\Psi}_{-}-\frac{1}{2 r}\left(1 \mp \sigma^{3}\right) \hat{\Psi}_{r} \\
-\frac{2 \sqrt{h}}{r} i \sigma^{1} \partial_{+} \hat{\Psi}-\frac{1}{\sqrt{h} r}\left(-i \sigma^{1}+\sigma^{2}\right) \partial_{-} \hat{\Psi}-\frac{1}{r} \sigma^{3} \partial_{r} \hat{\Psi}-\frac{h^{\prime}}{4 h r} \hat{\Psi}-\frac{7}{r^{2}}\left( \pm 1+\sigma^{3}\right) \hat{\Psi}=0
\end{array}
$$

We have 8 first-order equations (four 2-component equations) for $2 \times 4$ variables $\left(\hat{\Psi}_{+}, \hat{\Psi}_{-}\right.$, $\left.\hat{\Psi}_{r}, \hat{\Psi}\right)$. As is the case for Rarita-Schwinger field, $3 \times 2$ degrees of freedom are reduced by gauge fixing and constraints and remaining degrees of freedom are halved due to the Dirac constraint. Thus, there is one physical degree of freedom. We have obtained the Klein-Gordon-type 2nd-order equation for physical mode which should correspond to the result of elimination of unphysical variable from Dirac-type 1st-order equations.

The physical mode corresponding to $\Xi^{k=0,+}$ (the upper signs in the above equations) satisfies

$$
\left[-\partial_{t}^{2}+\partial_{z}^{2}+\frac{1}{z} \partial_{z}-\left(\frac{21}{5}\right)^{2} \frac{1}{z^{2}}\right]\left(z^{-7 / 2} \hat{\Psi}_{-}^{(1)}\right)=0
$$

where $\hat{\Psi}_{-}^{(1)}$ is the upper component of $\hat{\Psi}_{-}\left(\sigma^{3} \hat{\Psi}_{-}^{(1)}=+\hat{\Psi}_{-}^{(1)}\right)$. The derivation of (3.30) is presented in Appendix B. We remark here that we have done the reinterpretation $x^{+} \rightarrow 2 t$ and set $\partial_{-}=0$ which corresponds to considering the near-horizon limit in 10 dimensions. Of course, our choice of the physical degree of freedom $\left(\hat{\Psi}_{-}^{(1)}\right)$ is not unique for it can be expressed by (the combination of) other variables using the constraint equations. In addition, we have to note that the order of the Bessel function $\nu(=21 / 5$ in this case) is not unique. That is, when there is a field which satisfy Bessel equation with order $\nu$

$$
\left[-\partial_{t}^{2}+\partial_{z}^{2}+\frac{1}{z} \partial_{z}-\frac{\nu^{2}}{z^{2}}\right] \phi=0,
$$

The following combination satisfies the Bessel equation with the order shifted by \pm 1 . 目

$$
\left[-\partial_{t}^{2}+\partial_{z}^{2}+\frac{1}{z} \partial_{z}-\frac{(\nu \pm 1)^{2}}{z^{2}}\right]\left(\partial_{z} \phi \mp \frac{\nu}{z} \phi\right)=0 .
$$

This fact corresponds to the identity of the Bessel function

$$
z K_{\nu}^{\prime}(z) \pm \nu K_{\nu}(z)=-z K_{\nu \mp 1}(z)
$$

$\S$ The difference of the orders for the upper $\left(C^{(1)}\right)$ and the lower $\left(C^{(2)}\right)$ component of the vector-spinor mode $\hat{\Psi}$ in (3.21) is an example of this ambiguity of the order of the physical degree of freedom. 
In (3.30), we have chosen the representative for physical mode whose order $\nu$ allows interpretation in terms of the generalized AdS/CFT correspondence, as we shall see in section 4 .

As a result of a similar analysis for $\Xi^{k=0,-}$ mode (the lower signs in the field equations), we obtain the physical mode for this case

$$
\left[-\partial_{t}^{2}+\partial_{z}^{2}+\frac{1}{z} \partial_{z}-\left(\frac{14}{5}\right)^{2} \frac{1}{z^{2}}\right]\left(z^{-7 / 2} \Psi_{-}^{(2)}\right)=0 .
$$

where $\sigma^{3} \hat{\Psi}_{-}^{(2)}=-\hat{\Psi}_{-}^{(2)}$. The same remark as in the $\Xi^{k=0,+}$ case for the order of the Bessel function applies.

\subsection{Spinor modes at general level $(k=1,2, \ldots)$}

In this case, we analyze 5 equations (3.2) (3.6). Under the gauge condition $\Gamma^{i} \Psi_{i}=0$, the equations for $\Xi^{k, \pm}$ modes $(k=1,2, \ldots)$ are the following. The upper signs are for the $\Xi^{k,+}$ mode and the lower signs are for the $\Xi^{k,-}$ mode. From (3.2),

$$
\begin{gathered}
-2\left(\partial_{-} \hat{\Psi}_{r}-\partial_{r} \hat{\Psi}_{-}\right)+\frac{h^{\prime}}{2 h} \sigma^{3} \hat{\Psi}_{-}+\frac{2}{r}\left(\mp(k+4) \sigma^{3}+4\right) \hat{\Psi}_{-} \\
+\frac{h^{\prime}}{2 \sqrt{h}}\left(-i \sigma^{1}+\sigma^{2}\right) \hat{\Psi}_{r}-\frac{2 \sqrt{h}}{r}\left(\mp(k+4) \sigma^{2}+4 i \sigma^{1}\right) \hat{\Psi}_{r}+\frac{7}{4} k(k+8) \frac{\sqrt{h}}{r^{2}} i \sigma^{1} \hat{\hat{\Psi}}=0(3.3
\end{gathered}
$$

From (3.3),

$$
\begin{aligned}
& 2\left(\partial_{+} \hat{\Psi}_{r}-\partial_{r} \hat{\Psi}_{+}\right)-\frac{h^{\prime}}{2 h} \sigma^{3} \hat{\Psi}_{+}+\frac{2}{r}\left( \pm(k+4) \sigma^{3}-4\right) \hat{\Psi}_{+} \\
& +\frac{1}{r \sqrt{h}}(\mp(k+4)-4)\left(-i \sigma^{1}+\sigma^{2}\right) \hat{\Psi}_{r}+\frac{7}{8} k(k+8) \frac{1}{r^{2} \sqrt{h}}\left(-i \sigma^{1}+\sigma^{2}\right) \hat{\hat{\Psi}}=0
\end{aligned}
$$

From (3.4),

$$
\begin{aligned}
& -2 \sigma^{3}\left(\partial_{+} \hat{\Psi}_{-}-\partial_{-} \hat{\Psi}_{+}\right)-\frac{h^{\prime}}{2 \sqrt{h}}\left(-i \sigma^{1}+\sigma^{2}\right) \hat{\Psi}_{+}+\frac{2 \sqrt{h}}{r}\left( \pm(k+4) i \sigma^{1}-4 \sigma^{2}\right) \hat{\Psi}_{+} \\
& +\frac{1}{r \sqrt{h}}( \pm(k+4)+4)\left(-i \sigma^{1}+\sigma^{2}\right) \hat{\Psi}_{-}+\frac{7}{8} k(k+8) \frac{1}{r^{2}} \hat{\hat{\Psi}}=0
\end{aligned}
$$

From (3.5),

$$
\begin{aligned}
& 2 \sqrt{h}\left(\mp(k+4) i \sigma^{1}+4 \sigma^{2}\right) \hat{\Psi}_{+}+\frac{1}{\sqrt{h}}(\mp(k+4)-4)\left(-i \sigma^{1}+\sigma^{2}\right) \hat{\Psi}_{-} \\
& +\left(\mp(k+4) \sigma^{3}+4\right) \hat{\Psi}_{r}-\frac{7}{4} k(k+8) \frac{1}{r} \hat{\hat{\Psi}}=0
\end{aligned}
$$


From (3.6),

$$
\begin{aligned}
& -2 \sqrt{h} i \sigma^{1} \hat{\Psi}_{+}-\frac{1}{\sqrt{h}}\left(-i \sigma^{1}+\sigma^{2}\right) \hat{\Psi}_{-}-\sigma^{3} \hat{\Psi}_{r}+2 \sqrt{h} i \sigma^{1} \partial_{+} \hat{\hat{\Psi}} \\
& +\frac{1}{\sqrt{h}}\left(-i \sigma^{1}+\sigma^{2}\right) \partial_{-} \hat{\hat{\Psi}}+\sigma^{3} \partial_{r} \hat{\hat{\Psi}}+\frac{h^{\prime}}{4 h} \hat{\hat{\Psi}}+\frac{3}{4}\left(\mp(k+4)+4 \sigma^{3}\right) \frac{1}{r} \hat{\hat{\Psi}}=0
\end{aligned}
$$

There are two independent physical degrees of freedom. This is as expected, for the total number of degrees of freedom for $k \geq 1$ mode equals that of the $\mathrm{RS}$ field. That is, $2 \times 16$ for spinor modes $(\Xi)$ plus $(8-2) \times 16$ for vector-spinor modes $\left(\Xi_{i}\right)(-2$ for the conditions $\left.D^{i} \Xi_{i}=\gamma^{i} \Xi_{i}=0\right)$ equals the total degrees of freedom of RS fields $(11-3) \times 16$. We shall explain the outline of the calculation of the spectrum and present the results. First, we note that there are 5 sets of equations for 4 sets of variables. We can see that the system of the equations is consistent by noticing that (3.39) is obtained by taking a linear combination of derivatives of other four equations. Thus, we consider only the first four of the equations. Setting $\partial_{-}=0$ (and reinterpreting $x^{+} \rightarrow 2 t$ ), we can eliminate $\hat{\Psi}_{i}$, $\hat{\Psi}_{r}$ and $\hat{\Psi}_{+}$algebraically using (3.38), (3.37) and (3.35) and obtain the following equation for $\hat{\Psi}_{-}$.

$$
\begin{aligned}
& -\frac{2}{7} h \partial_{t}^{2} \hat{\Psi}_{-}+\frac{2}{7} \partial_{r}^{2} \hat{\Psi}_{-}+\left\{\frac{32}{7}+\left(\mp \frac{4}{7}(k+4)-1\right) \sigma^{3}\right\} \frac{1}{r} \partial_{r} \hat{\Psi}_{-} \\
& +\left\{\frac{2}{7}(k+4)^{2} \pm(k+4)+\frac{753}{56}+\left(\mp \frac{30}{7}(k+4)-\frac{15}{2}\right) \sigma^{3}\right\} \frac{1}{r^{2}} \hat{\Psi}_{-}+\frac{\left(\mp(k+4)+4 \sigma^{3}\right)}{k(k+8)} \\
& \cdot\left\{\left(\frac{8}{7}(k+4)^{2} \pm 2(k+4)-\frac{58}{7}\right) i \sigma^{1}+(\mp 2(k+4)-10)\right\} \frac{\sqrt{h}}{r} \partial_{+} \hat{\Psi}_{-}=0
\end{aligned}
$$

After diagonalizing this second-order coupled equations for two variables $\left(\hat{\Psi}_{-}^{(1)}\right.$ and $\hat{\Psi}_{-}^{(2)}$ where $\sigma^{3} \hat{\Psi}_{-}^{(1)}=+\hat{\Psi}_{-}^{(1)}$ and $\left.\sigma^{3} \hat{\Psi}_{-}^{(2)}=-\hat{\Psi}_{-}^{(2)}\right)$, we found two physical modes which are solved by the modified Bessel functions of the following order $\nu$. For $\Xi^{k,+}$ modes,

$$
\begin{array}{rlrl}
\nu_{1} & =\frac{2}{5} k+\frac{21}{5} & (k=0,1, \ldots), \\
\nu_{2}=\frac{2}{5} k+\frac{7}{5} & (k=1,2, \ldots) .
\end{array}
$$

where we have included the case $k=0$ in the formula. Explicit forms of the diagonalized fields $\varphi_{1}^{k,+}$ and $\varphi_{2}^{k,+}$ corresponding to $\nu_{1}$ and $\nu_{2}$ respectively are given (for $k \geq 1$ ) by

$$
\varphi_{1}^{k,+}=2 i \frac{(k+8)}{(2 k+9)} z^{-5 / 2}\left\{\partial_{z} \hat{\Psi}_{-}^{(1)}+\left(\frac{2}{5} k-\frac{21}{10}\right) \frac{1}{z} \hat{\Psi}_{-}^{(1)}\right\}+z^{-5 / 2} \partial_{t} \hat{\Psi}_{-}^{(2)}
$$


and

$$
\varphi_{2}^{k,+}=2 i \frac{(k+8)}{(2 k+9)} z^{-5 / 2}\left\{\partial_{z} \hat{\Psi}_{-}^{(1)}-\frac{\left(\frac{31}{10} k+\frac{119}{5}\right)}{(k+8) z} \hat{\Psi}_{-}^{(1)}\right\}+z^{-5 / 2} \partial_{t} \hat{\Psi}_{-}^{(2)} .
$$

For $\Xi^{k,-}$ modes, the orders of the Bessel functions are

$$
\begin{aligned}
\nu_{1}=\frac{2}{5} k & (k=1,2, \ldots), \\
\nu_{2}=\frac{2}{5} k+\frac{14}{5} & (k=0,1, \ldots) .
\end{aligned}
$$

Explicit forms of the diagonalized fields $\left(\varphi_{1}^{k,-}\right.$ and $\left.\varphi_{2}^{k,-}\right)$ for $k \geq 1$ are obtained from the following expressions.

$$
\begin{aligned}
& \tilde{\varphi}_{1}^{k,-}=\frac{2 i k}{(2 k+7)} z^{-5 / 2}\left\{\partial_{z} \hat{\Psi}_{-}^{(1)}-\left(\frac{2}{5} k+\frac{53}{10}\right) \frac{1}{z} \hat{\Psi}_{-}^{(1)}\right\}+z^{-5 / 2} \partial_{t} \hat{\Psi}_{-}^{(2)}, \\
& \tilde{\varphi}_{2}^{k,-}=\frac{2 i k}{(2 k+7)} z^{-5 / 2}\left\{\partial_{z} \hat{\Psi}_{-}^{(1)}-\frac{\left(\frac{31}{10} k+1\right)}{k z} \hat{\Psi}_{-}^{(1)}\right\}+z^{-5 / 2} \partial_{t} \hat{\Psi}_{-}^{(2)}
\end{aligned}
$$

$\tilde{\varphi}_{1}^{k,-}$ and $\tilde{\varphi}_{2}^{k,-}$ in $(3.45)$ are the solutions with orders $\tilde{\nu}_{1}=2 k / 5-1$ and $\tilde{\nu}_{2}=2 k / 5+9 / 5$ respectively which are related to the orders $\nu$ given in (3.44) by $\nu=\tilde{\nu}+1$. Thus, we can construct the solutions with order $\nu$ using the method described in the last subsection. The orders $\nu$ of (3.44) are the ones which allow the interpretations in terms of the generalized AdS/CFT correspondence.

\section{Generalized AdS/CFT Correspondence}

\subsection{Matrix-theory correlators form supergravity}

As we did in the case of bosons, we shall calculate the correlators in Matrix theory by evaluating the supergravity action. First, we recall in what situations the calculation of classical supergravity is valid [11]. The curvature of the D0-brane background must be small in string unit which gives

$$
r \ll\left(g_{s} N\right)^{1 / 3} \ell_{s}
$$

and the effective string coupling (dilaton background) must be small

$$
g_{s}^{1 / 3} N^{1 / 7} \ell_{s} \ll r .
$$

`Different expressions for $\varphi_{1}^{k,-}, \varphi_{2}^{k,-}$ having the orders (3.44) may be possible by using (3.40) again. 
Those two conditions are simultaneously satisfied in the whole near-horizon region

$$
r \ll\left(g_{s} N\right)^{1 / 7} \ell_{s},
$$

if

$$
N \rightarrow \infty, \quad\left(g_{s} N\right)>1 .
$$

We assume the following relation between the supergravity action and the generating functional of the Matrix-theory correlators.

$$
\mathrm{e}^{-S_{S G}[\Psi]}=\left\langle\exp \left\{\int d t \sum_{I} \Psi_{I}(t) \mathcal{O}_{I}(t)\right\}\right\rangle,
$$

where we impose the boundary conditions on the physical modes of supergravity at the end of the near-horizon region $\left(z=q^{1 / 7}\right.$ i.e. $\left.r \propto\left(g_{s} N\right)^{1 / 7} \ell_{s}\right)$ and evaluate the action on the near-horizon limit of the D0-brane background as a functional of the boundary conditions. In this paper, we obtain the two-point functions of the fermionic operators of Matrix theory.

We first analyze the transverse vector-spinor mode with the ' + ' choice of the eigenvalue of the Dirac operator on the sphere $\left(\Xi_{i}{ }^{(k,+)}\right.$ mode) and then present the results for general modes. The relevant part of the 10-dimensional action becomes (up to a numerical factor,)

$$
S=-i \frac{1}{\kappa^{2}} \int d t d r r^{8} \sum_{I} \hat{\Psi}^{I \dagger}\left(i \sigma^{1}\right)\left\{i \sigma^{1} \sqrt{h} \partial_{t}+\sigma^{3} \partial_{r}+\cdots\right\} \hat{\Psi}^{I} .
$$

We have used the orthonormality of the spherical harmonics

$$
\int d \Omega_{8} g^{i j} \Xi_{i}^{I^{\dagger}} \Xi_{j}^{J}=C \delta^{I J}
$$

where $C$ is a numerical constant and $I, J$ are the labels for the harmonics. Also note that we have chosen the normalization ( $g_{s}$ dependence) of the fields such that the action in 10 dimensions has $\kappa^{2} \sim 1 / g_{s}^{2} \ell_{s}^{8}$ as a prefactor.

Euclidizing the time coordinate $(t=-i \tau)$ and using the radial coordinate $z=$ $2 q^{1 / 2} r^{-5 / 2} / 5$,

$$
S=\frac{1}{\kappa^{2}} \int d \tau d z q^{8 / 5} z^{-16 / 5} \sum_{I} \hat{\Psi}^{I \dagger}\left(\sigma^{1}\right)\left\{\sigma^{1} \partial_{\tau}+\sigma^{3} \partial_{z}+\cdots\right\} \hat{\Psi}^{I}
$$


As noted in section 3.3, we can impose the boundary condition to one of the two components of $\hat{\Psi}$. Following the choice which is assumed in the case of ordinary AdS/CFT correspondence [19], we fix the component which is more divergent as we take the boundary to infinity $(z \rightarrow 0)$. That is, we fix $\hat{\Psi}^{(1)}$ for the $\Xi_{i}^{k,+} \operatorname{mode}\left(\right.$ by noting $K_{\nu}(z) \sim z^{-\nu}$ ). For the same reason, we fix $\hat{\Psi}^{(2)}$ in the case of the $\Xi_{i}^{k,-}$ mode. The action is of first order and the bulk contribution vanishes on shell, but we need to add the following boundary term (at $\left.z=q^{1 / 7}\right)$ to the action

$$
S=S_{\text {boundary }}=-\frac{1}{2 \kappa^{2}} q^{8 / 5-16 / 35} \int d \tau \hat{\Psi}^{\dagger} \sigma^{1} \hat{\Psi}
$$

to ensure that the classical solution is really an extremum of the action when we fix $\hat{\Psi}^{(1)}$ at the boundary [20].

Substituting the solution for the $\Xi_{i}^{(k,+)}$ mode

$$
\hat{\Psi}=\int \frac{d \omega}{2 \pi} \mathrm{e}^{-i \omega \tau}\left(z / q^{1 / 7}\right)^{21 / 10}\left\{K_{\frac{2}{5}\left(k+\frac{7}{2}\right)}(|\omega| z)+\frac{i \omega}{|\omega|} \sigma^{1} K_{\frac{2}{5}(k+1)}(|\omega| z)\right\} \frac{\hat{\Psi}_{b}^{(1)}(\omega)}{K_{\frac{2}{5}\left(k+\frac{7}{2}\right)}\left(|\omega| q^{1 / 7}\right)}
$$

which satisfy boundary condition $\hat{\Psi}^{(1)}\left(\tau, z=q^{1 / 7}\right)=\int d \omega \mathrm{e}^{-i \omega \tau} \hat{\Psi}_{b}^{(1)}(\omega) / 2 \pi$, the action reads

$$
\begin{aligned}
S & =\frac{1}{\kappa^{2}} q^{8 / 7} \int \frac{d \omega}{2 \pi} \hat{\Psi}_{b}^{(1)}(-\omega) \hat{\Psi}_{b}^{(1)}(\omega) \frac{i \omega}{|\omega|} \frac{K_{\nu-1}\left(|\omega| q^{1 / 7}\right)}{K_{\nu}\left(|\omega| q^{1 / 7}\right)} \\
& =2^{-2 \nu+1} \frac{\Gamma(1-\nu)}{\Gamma(\nu)} \frac{1}{\kappa^{2}} q^{1+2 \nu / 7} \int \frac{d \omega}{2 \pi} \hat{\Psi}_{b}^{(1)}(-\omega) \hat{\Psi}_{b}^{(1)}(\omega) i \omega\left\{|\omega|^{2 \nu-2}+\cdots\right\}
\end{aligned}
$$

where

$$
\nu=\frac{2}{5} k+\frac{7}{5}
$$

and we have retained only the leading part which is non-analytic in $\omega$. We have used the Majorana condition (for our representation, $\hat{\Psi}^{\dagger}=\hat{\Psi}^{T} \sigma^{3}$ ).

Following the ansatz of the generalized AdS/CFT correspondence (4.5), we obtain the two-point function of the operator $\mathcal{O}$ which couple to the mode $\Psi^{(1)}$

$$
\begin{aligned}
\langle\mathcal{O}(\omega) \mathcal{O}(-\omega)\rangle & =-\frac{\delta}{\delta \hat{\Psi}_{b}^{(1)}(-\omega)} \frac{\delta}{\delta \hat{\Psi}_{b}^{(1)}(\omega)} S_{S G} \\
& =-\frac{2^{-2 \nu+1}}{\pi} \frac{\Gamma(1-\nu)}{\Gamma(\nu)} \frac{1}{\kappa^{2}} q^{1+2 \nu / 7} i \omega\left\{|\omega|^{2 \nu-2}+\cdots\right\} .
\end{aligned}
$$


Fourier transforming to the position space,

$$
\begin{aligned}
\left\langle\mathcal{O}\left(\tau_{1}\right) \mathcal{O}\left(\tau_{2}\right)\right\rangle & =\int \frac{d \omega}{2 \pi} \mathrm{e}^{-i \omega \tau} \int \frac{d \omega^{\prime}}{2 \pi} \mathrm{e}^{-i \omega^{\prime} \tau}\left\langle\mathcal{O}(\omega) \mathcal{O}\left(\omega^{\prime}\right)\right\rangle \\
& =\frac{1}{2 \pi^{-3 / 2}} \frac{\Gamma(\nu+1 / 2)}{\Gamma(\nu)} \frac{1}{\kappa^{2}} q^{1+2 \nu / 7} \frac{\left(\tau_{1}-\tau_{2}\right)}{\left|\tau_{1}-\tau_{2}\right|^{2 \nu+1}}
\end{aligned}
$$

The 2-point functions corresponding to the other physical modes are given by substituting into (4.13) the order $\nu$ obtained in sections 3.3, 3.4 and 3.5.

\subsection{Identification of the Matrix-theory operators}

Following the generalized AdS/CFT correspondence (4.5), we have calculated the 2-point functions of Matrix-theory operators which couple to Rarita-Schwinger fields of the 11dimensional supergravity. Now we would like to identify the operators which couple to each physical mode of supergravity. RS fields are the gauge fields of the supersymmetry and should couple to the supercurrents of Matrix theory, but we do not have principles which determine the coupling. We shall determine the Matrix-theory operator by requiring 1) the scaling dimension (with respect to the generalized conformal symmetry which is explained below) agrees with the one read off from the correlator which was obtained form the supergravity calculation, and 2) the representation with respect to the transverse rotation group $\mathrm{SO}(9)$ agrees with the supergravity mode. Note that this situation is essentially the same as in the ordinary AdS/CFT correspondence such as the $A d S_{5} / C F T_{4}$ case, where the representation with respect to the $\mathcal{N}=4$ superconformal symmetry is the guideline for determining the coupling.

Matrix theory is defined by the following action.

$$
S=\frac{1}{g_{s} \ell_{s}} \int d t \operatorname{Tr}\left(\frac{1}{2} D_{t} X^{m} D_{t} X^{m}+\frac{i}{2} \theta D_{t} \theta+\frac{1}{4 \ell_{s}^{4}}\left[X^{m}, X^{n}\right]^{2}-\frac{1}{2 \ell_{s}^{2}} \theta \gamma^{m}\left[\theta, X^{m}\right]\right)
$$

where $D_{t}=\partial_{t}+i[A, \quad]$. and $\gamma^{m}$ are the 9-dimensional Gamma matrices which are real and symmetric. It has the generalized conformal symmetry which allows the coupling constant $g_{s}$ to be transformed. The scale transformation is given by

$$
t \rightarrow \lambda^{-1} t, \quad X_{m} \rightarrow \lambda X_{m}, \quad \theta \rightarrow \lambda^{3 / 2} \theta, \quad g_{s} \rightarrow \lambda^{3} g_{s}
$$


Together with the time translation and the special conformal transformation (treating as $g_{s}$ as a field with dimension:3), they constitute the conformal algebra. The near-horizon limit of the D0-brane solution has the same kind of symmetry [10].

Matrix theory has two kinds of fermionic symmetries. One is a constant shift of fermionic field only.

$$
\delta \theta=\tilde{\epsilon}
$$

The other is the usual supersymmetry of the SYM theory.

$$
\begin{aligned}
& \delta X^{m}=i \epsilon^{T} \gamma^{m} \theta, \quad \delta A=i \epsilon^{T} \theta, \\
& \delta \theta=-\frac{1}{2}\left(2 D_{t} X_{m} \gamma^{m}+\frac{i}{\ell_{s}^{2}}\left[X_{m}, X_{n}\right] \gamma^{m n}\right) \epsilon
\end{aligned}
$$

The explicit forms of the supercurrents of Matrix theory are obtained by Taylor and van Raamsdonk [21]. They are identified by reinterpreting the one-loop effective potential between a pair of diagonal blocks in Matrix theory as the potential between the currents in the linearized supergravity compactified on $x^{-}$. The $(M=+)$ and $(M=m)$ components of the supercurrents are given as follows up to numerical constant factors and possible three-fermion terms. The currents are assumed to be integrated in the $x^{-}$-direction.

$$
\begin{aligned}
\tilde{q}^{+} & =\frac{1}{R} \operatorname{Tr}(\theta) \\
\tilde{q}^{m} & =\frac{1}{R} \operatorname{Tr}\left(\left\{\dot{X}^{m}-F_{m n} \gamma^{n}\right\} \theta\right) \\
q^{+} & =\frac{1}{R} \operatorname{Tr}\left(\left\{\dot{X}^{m} \gamma_{m}+\frac{1}{2} F_{m n} \gamma^{m n}\right\} \theta\right) \\
q^{m} & =\frac{1}{R} \operatorname{STr}\left(\left\{2 \dot{X}^{m} \dot{X}^{n} \gamma_{n}+\dot{X}^{m} F_{n p} \gamma^{n} \gamma^{p}+2 \dot{X}_{n} F_{p m} \gamma^{n} \gamma^{p}+F_{n p} F_{q m} \gamma^{n} \gamma^{p} \gamma^{q}\right\} \theta\right)
\end{aligned}
$$

where $F_{m n}=i\left[X_{m}, X_{n}\right]$ and $\mathrm{STr}$ is the symmetrized trace in which the average is taken over all possible orderings of matrices (treating $F_{m n}$ as a single unit). The above expression is for the $A=0$ gauge and we have set $\ell_{s}=1$. Note that $\tilde{q}^{+}$and $q^{+}$agree with the supercharges in the SYM theory corresponding to (4.16) and (4.17). The $(M=-)$ components of the supercurrents are of the form (up to 3-fermion terms)

$$
\begin{aligned}
& \tilde{q}^{-}=\frac{1}{R} \operatorname{STr}\left(\left\{\text { terms of } 2 \text { nd order in } F_{m n}, \dot{X}_{m}\right\} \theta\right) \\
& q^{-}=\frac{1}{R} \operatorname{STr}\left(\left\{\text { terms of } 3 \text { rd order in } F_{m n}, \dot{X}_{m}\right\} \theta\right)
\end{aligned}
$$


To obtain the explicit forms of $\tilde{q}^{-}$and $q^{-}$, analysis of the ' $1 / r^{8}$ terms' of Matrix-theory effective potential is needed. (Terms in (4.18) are obtained from the ' $1 / r^{7}$ terms'.) In addition to the operators (4.18) and (4.19), there are the 'moments' of the currents which couple to the transverse derivatives of the supergravity fields. The $k$-th moment $q_{(k)}^{M\left\{m_{1}, \ldots, m_{k}\right\}}$, $\tilde{q}_{(k)}^{M\left\{m_{1}, \ldots, m_{k}\right\}}$ contain a term which is obtained by inserting $k$ times the transverse field $\tilde{X}^{m}=X^{m} / q^{1 / 7}$ into the traces in (4.18) and (4.19).

The consistency of these couplings between the branes and the supergravity fields are confirmed in various contexts: they are consistent with the couplings obtained by expanding the Matrix-regularized membrane action in the lightcone gauge around the flat background [22]; the couplings for D0-branes which are obtained through the Seiberg-Sen argument are consistent with the Born-Infeld action [23]; the couplings for D $p$-branes obtained from D0 couplings by T-duality indeed have $(p+1)$-dimensional Lorentz covariance [24]; and notably, the leading part of the absorption cross sections by D3-branes in the small $\left(g_{s} N\right)$, large $N$ limit which is calculated in the $D=4, \mathcal{N}=4$ SYM theory using the T-dualized couplings exactly reproduce the ones calculated in the semiclassical supergravity including the cases of higher partial waves [25].

We assume that these operators are the candidates for the operators which couple to the supergravity modes in our case. We shall compare the scaling dimensions (with respect to the generalized conformal symmetry (4.15)) of the supercurrents and that of the correlators obtained in the last subsection. From the right hand side of (4.13), we see that the scaling dimension $\Delta$ of the operator is

$$
\begin{aligned}
\Delta & =\frac{1}{2}\left\{3 \cdot(-2)+3 \cdot\left(1+\frac{2 \nu}{7}\right)+2 \nu\right\} \\
& =-\frac{3}{2}+\frac{10}{7} \nu
\end{aligned}
$$

We can consistently identify the Matrix-theory operator which have the dimension (4.20) for every supergravity modes. The results are summarized in the Table below. Operators $q_{(k)}^{m}$ and $\tilde{q}_{(k)}^{m}$ in the Table are the ones which have the same $\mathrm{SO}(9)$ quantum numbers as the harmonics. e.g., $\tilde{q}_{(1)}^{m\left\{m_{1}\right\}} \sim \frac{1}{R} \operatorname{STr}\left\{\left(\dot{X}^{m}-F_{m n} \gamma^{n}\right) X^{m_{1}} \theta\right\}-\left(m \leftrightarrow m_{1}\right)$. 


\section{Table}

Physical modes of supergravity and the corresponding Matrix-theory operators

\begin{tabular}{|c||c|c|c|c|c|c|}
\hline \multicolumn{1}{|c||}{ SUGRA fields } & \multicolumn{2}{c|}{$\Psi_{i}$} & \multicolumn{4}{c|}{$\Psi_{+}, \Psi_{-}, \Psi_{r}, \Psi_{i}$} \\
\hline harmonics & $\Xi_{i}^{k,+}$ & $\Xi_{i}^{k,-}$ & $\Xi^{k,+}$ & $\Xi^{k,+}$ & $\Xi^{k,-}$ & $\Xi^{k,-}$ \\
\hline physical modes & $z^{-7 / 2} \hat{\Psi}^{(1)}$ & $z^{-7 / 2} \hat{\Psi}^{(2)}$ & $\varphi_{1}^{k,+}$ & $\varphi_{2}^{k,+}$ & $\varphi_{2}^{k,-}$ & $\varphi_{1}^{k,-}$ \\
\hline order $\nu$ & $\frac{2}{5} k+\frac{7}{5}$ & $\frac{2}{5} k+\frac{14}{5}$ & $\frac{2}{5} k+\frac{21}{5}$ & $\frac{2}{5} k+\frac{7}{5}$ & $\frac{2}{5} k+\frac{14}{5}$ & $\frac{2}{5} k$ \\
\hline regions of $k$ & $k \geq 1$ & $k \geq 1$ & $k \geq 0$ & $k \geq 1$ & $k \geq 0$ & $k \geq 1$ \\
\hline operator $\mathcal{O}$ & $\tilde{q}_{(k)}^{m}$ & $q_{(k)}^{m}$ & $q_{(k)}^{-}$ & $q_{(k)}^{+}$ & $\tilde{q}_{(k)}^{-}$ & $\tilde{q}_{(k)}^{+}$ \\
\hline dimensions of $\mathcal{O}$ & $\frac{1}{2}+\frac{4}{7} k$ & $\frac{5}{2}+\frac{4}{7} k$ & $\frac{9}{2}+\frac{4}{7} k$ & $\frac{1}{2}+\frac{4}{7} k$ & $\frac{5}{2}+\frac{4}{7} k$ & $-\frac{3}{2}+\frac{4}{7} k$ \\
\hline
\end{tabular}

\section{11-dimensional Lorentz invariance and Supersymmetry of Matrix the- ory}

In this section we consider the implications of our results for the Matrix-theory conjecture, following the line of arguments of the previous paper [11]. Some of the results of [11] are reviewed for completeness. According to the conjecture of Banks, Fischler, Shenker and Susskind, infinite momentum frame $\left(P_{-} \rightarrow \infty\right)$ is achieved by taking the limit

$$
N \rightarrow \infty \quad \text { with } \quad g_{s} \text { : fixed. }
$$

In this region, Matrix theory is expected to have the boost invariance in the $x^{11}$ direction, which rescales $N$ with fixed $g_{s}$. As argued in section 4.1, our results obtained from supergravity is valid when $N \rightarrow \infty$ and $\left(g_{s} N\right)>1$, which will allows us to investigate the infinite momentum limit. We shall check the boost invariance in the sense of the large- $N$ scaling behavior of the operators.

Under the boost transformation

$$
x^{+} \rightarrow \mathrm{e}^{\omega} x^{+}, \quad x^{-} \rightarrow \mathrm{e}^{-\omega} x^{-},
$$

where $P_{-}$scales as $N$, the time coordinate of Matrix theory which is the lightcone time scales as $N$, Matrix-theory Hamiltonian $\left(P_{+}\right)$must scale as $1 / N$ while the transverse coordinates and the compactification radius are kept fixed. We shall analyze the behavior of

\footnotetext{
"Strictly speaking, the condition for the validity of supergravity (4.4) is different from the infinite momentum limit (5.1). To satisfy (4.4), we need to take $g_{s} \rightarrow 0$ as $N \rightarrow \infty$ rather than fixing $g_{s}$. We are assuming that this difference does not affect the results.
} 
the operators under the boost from the scaling behavior of the correlators. We substitute $\tau \rightarrow N \tau$ in the correlators and examine the $N$ dependence.

$$
\left\langle\mathcal{O}\left(\tau_{1}\right) \mathcal{O}\left(\tau_{2}\right)\right\rangle \rightarrow N^{2 d_{I M F}} G\left(\tau_{1}-\tau_{2}\right)
$$

where $G(\tau)$ is a function independent of $N$ and we have defined the 'scaling dimension with respect to the boost transformation' $d_{I M F}$ of the operator $\mathcal{O}$.

First, we review the behavior of the correlators of the bosonic operators. From the generalized AdS/CFT correspondence we have obtained [11]

$$
\left\langle\mathcal{O}\left(\tau_{1}\right) \mathcal{O}\left(\tau_{2}\right)\right\rangle=\frac{1}{g_{s}^{2} \ell_{s}^{8}}\left(g_{s} N \ell_{s}^{7}\right)^{1+2 \nu / 7} \frac{1}{\left|\tau_{1}-\tau_{2}\right|^{2 \nu+1}} .
$$

where $\nu$ is the order of the modified Bessel function of the corresponding supergravity mode. By examining the values of $\nu$, we have found that the 'dimension' $d_{I M F}$ is given by

$$
d_{I M F}=\frac{6}{7} \nu=\left(1+\frac{1}{5}\right)\left(n_{+}-n_{-}-1\right)-\left(\frac{1}{7}+\frac{1}{5}\right) k .
$$

where $n_{+}\left(n_{-}\right)$is the number of the upper $+(-)$index on the operator and $k$ means the $k$ th moment. The fact that $d_{I M F}$ is determined solely from the spacetime index structure of the operators suggests that the scaling of $N$ is indeed related to the spacetime symmetry. The tensor with the + index indeed scales inversely as the tensor with the - index. However, the weights $\pm 6 / 5$ are different from the natural expectation \pm 1 by $\pm 1 / 5$. The interpretation of the remaining terms are as follows. The $k$-th moment has a contribution $-(1 / 7+1 / 5) k$. The factor $-1 / 7$ is explained by the fact that the moments which we are considering are made by multiplying $\tilde{X}^{m} \sim X^{m} /\left(\left(g_{s} N\right)^{1 / 7} \ell_{s}\right.$ ) (but not $\left.X^{m}\right) k$ times, but there is also an anomalous behavior $-1 / 5$ for each transverse field. The constant part $(-(1+1 / 5))$ is interpreted as coming from the $x^{-}$integration when we define the currents, also with the anomalous behavior for $x^{-}(-1 / 5)$.

Now we shall analyze the scaling behavior of the supercurrents. From the correlator obtained in the last section

$$
\left\langle\mathcal{O}\left(\tau_{1}\right) \mathcal{O}\left(\tau_{2}\right)\right\rangle=\frac{1}{g_{s}^{2} \ell_{s}^{8}}\left(g_{s} N \ell_{s}^{7}\right)^{1+2 \nu / 7} \frac{\left(\tau_{1}-\tau_{2}\right)}{\left|\tau_{1}-\tau_{2}\right|^{2 \nu+1}},
$$

we find the following scaling properties of the fermionic operators of Matrix theory.

$$
d_{I M F}=\frac{1}{2}-\frac{6}{7} \nu
$$




$$
=\left(1+\frac{1}{5}\right)\left(n_{+}-n_{-}-1\right)-\left(\frac{1}{7}+\frac{1}{5}\right) k \mp \frac{1}{2}\left(1+\frac{1}{5}\right)-\frac{1}{10}
$$

where the $(-)$ sign on $\frac{1}{2}\left(1+\frac{1}{5}\right)$ is for the current $q^{M}$ and the $(+)$ sign is for the current $\tilde{q}^{M}$. The interpretations of the scaling for the lightcone indices, for $x^{-}$-integration and for the moments are the same as in the bosonic case. In addition, we see that $q^{M}$ and $\tilde{q}^{M}$ transform inversely. This is indeed needed for spacetime interpretation. The current $\tilde{q}^{M}$ is the supercurrent corresponding to the constant shift of fermionic field (4.16) in Matrix theory, which is interpreted as the kinematical SUSY in 11 dimensions. The current $q^{M}$ is the supercurrent corresponding to the supersymmetry of the SYM theory (4.16), which is interpreted as the dynamical SUSY in 11 dimensions. The kinematical supercharge $(\tilde{q})$ satisfy $\Gamma^{-} \tilde{q}=0$ and the dynamical supercharge $(q)$ satisfy $\Gamma^{+} q=0$ to form the lightcone SUSY algebra $\left(\{\tilde{q}, \tilde{q}\} \sim P_{-} \Gamma^{-},\{\tilde{q}, q\} \sim P_{m} \Gamma^{m},\{q, q\} \sim P_{+} \Gamma^{+}\right)$. Under the boost (5.2), the kinematical and dynamical SUSY charges transform as

$$
\tilde{q} \rightarrow \mathrm{e}^{-\omega \Gamma^{0,11} / 2} \tilde{q}=\mathrm{e}^{\omega / 2} \tilde{q}, \quad q \rightarrow \mathrm{e}^{-\omega \Gamma^{0,11} / 2} q=\mathrm{e}^{-\omega / 2} q
$$

which can be shown from the properties of $q, \tilde{q}$ mentioned in the above sentences. Thus we expect that the current $\tilde{q}^{M}$ has a contribution $-1 / 2$ for the boost weight, and the current $q^{M}$ has $+1 / 2$. Our results shows anomalous behavior $\pm 1 / 10$ also in this case. We do not have an interpretation for the last term $-1 / 10$ of (5.7).

The interpretation of the anomalous behavior is not clear. One possibility is that Matrix theory does not have 11-dimensional Lorentz invariance or supersymmetry, but the following interpretation seems more appropriate [11]. We have studied Matrix theory by analyzing the supergravity inside the near-horizon region. However, it is possible that we could not take into account the necessary degrees of freedom of Matrix theory by restricting to the near-horizon region. Indeed, 'average transverse size' of Matrix theory $L^{2} \equiv \operatorname{Tr}\left(X^{m} X^{m}\right) / N$ is estimated to be $L \sim\left(g_{s} N\right)^{1 / 3} \ell_{s}$ [26], which is larger than the 'boundary' of the near-horizon region $r_{b} \sim\left(g_{s} N\right)^{1 / 7} \ell_{s}$ in the case of interest $\left(g_{s} N\right)>1$. Further argument supporting this interpretation was given in 27. Suppose we shift the boundary $r_{b} \sim N^{1 / 7}$ to some larger value $\tilde{r}_{b} \sim N^{\alpha}$. Assuming that the system is homogeneous (though it will not really be the case), the field $X^{m}$ will be rescaled to $\frac{\tilde{r}_{b}}{r_{b}} X^{m}$. If we require the factor just cancels the anomalous behavior for transverse fields 
$N^{-1 / 5}$, the shifted boundary must be

$$
\tilde{r}_{b} \propto N^{1 / 7+1 / 5}=N^{1 / 3+1 / 105}
$$

which is very close to $L \sim N^{1 / 3}$. It seems to suggest that we need to have radial size at least $L \sim\left(g_{s} N\right)^{1 / 3} \ell_{s}$ to analyze Matrix theory.

\section{Discussions}

In this paper, we have predicted the 2-point functions of the supercurrents of Matrix theory following the conjecture of generalized AdS/CFT correspondence. We have analyzed the scaling behavior with respect to $N$ and considered whether the Matrix-theory supercurrents have correct weights when we interpret the scaling of $N$ (with fixed $g_{s}$ ) as the boost in the 11-th direction following the original proposal of Banks, Fischler, Shenker and Susskind [1]. We have reached to a result which is not very definitive: The supercurrents corresponding to the two kinds of fermionic symmetries of Matrix theory can be indeed interpreted as having inverse weights under the Lorentz boost, which suggests the correctness of the interpretation of them as kinematical and dynamical SUSY currents of the 11-dimensional SUSY. However, the weights are slightly different from the canonical behavior, which seem to be of the same nature as the anomalous behavior for the spacetime tensors which were found in the previous paper. The origin of these behavior are not clear but it is likely that the generalized AdS/CFT correspondence is not able to give the exact information of Matrix theory, possibly due to the 'IR cutoff' of the supergravity calculation which is the boundary of the near-horizon region.

To understand the effect of this cutoff more precisely, we will have to study the cutoff dependence from both supergravity and gauge theory. On the supergravity side, one

possibility is to try to extend further the generalized AdS/CFT correspondence assuming that the bulk/boundary correspondence continues to hold for outside the near-horizon region. The holographic renormalization group [28] is a suitable framework for analyzing the effect of shifting the position of the boundary. On the gauge-theory side, on the other hand, analyzing the dependence on the cutoff (for transverse fields $X^{m}$ ) may be as difficult as solving the theory directly. Up to now, quantitative study of the large- $N$ Matrix theory 
in the strong coupling is only attempted through the Gaussian approximation [29, 30]. We hope that the cutoff dependence can be incorporated in some approximation scheme.

Though we have mentioned the possibility for the limitation of the generalized AdS/CFT correspondence, our results which exhibit the large- $N$ scaling behavior should be the consequence of the collective dynamics of Matrix theory. Though the near-horizon region $r<\left(g_{s} N\right)^{1 / 7} \ell_{s}$ is smaller than the average transverse size $L \sim\left(g_{s} N\right)^{1 / 3} \ell_{s}$, it should be noted that it is larger than the characteristic size of $N$-body bound state obtained by a mean field analysis [1] $L_{m . f .} \sim\left(g_{s} N\right)^{1 / 9} \ell_{s}$. We would like to note a fact which may be an indication [1] that the generalized AdS/CFT correspondence takes into account certain amounts of degrees of freedom of Matrix theory. Assume that the coefficient of the twopoint function of the stress-energy tensor gives the entropy of the theory. The coefficient (c) of the two-point function of $T_{i j}$ in the lowest angular momentum $(k=0)$ mode which is obtained from the generalized AdS/CFT correspondence $c \propto N^{2}\left(g_{s} N\right)^{-3 / 5}$ agrees with the $g_{s}$ and $N$ dependence of the entropy which is calculated from the Bekenstein-Hawking formula $S \propto N^{2}\left(g_{s} N \ell_{s}^{3} / T_{H}^{3}\right)^{-3 / 5}$ where $T_{H}$ is the Hawking temperature. We hope to clarify the meaning of this agreement in future works.

Once the nature and the validity of the generalized AdS/CFT correspondence for Matrix theory are understood, we can use this formalism to investigate other dilatonic Dbranes, for the general $\mathrm{D} p$-branes have the generalized conformal symmetry. Particularly interesting case is that of D1-branes. The $\mathrm{U}(N)$ super Yang-Mills theory in 2 dimensions describing D1-branes is called Matrix string theory and is conjectured to be another nonperturbative definition of the type IIA string theory [31]. In the large- $N$ limit with small $g_{s}$, this theory is believed to be described effectively by a conformal field theory, and an effective interaction vertex operator is proposed which is claimed to have leading scaling dimension. The generalized AdS/CFT correspondence for D1-branes will be useful in checking these conjectures and in investigating the non-perturbative behavior of string theory.

\section{Acknowledgement}

I would like to thank T. Yoneya for valuable discussions at every stage of this work and for careful reading of the manuscript. I also thank W. Taylor for reading the manuscript 
and for giving important comments.

\section{Appendix}

\section{A. The connections and the curvatures of the background}

We summarize here the connections and the curvatures of the background in 11 dimensions which is given by the metric

$$
d s^{2}=d x^{+} d x^{-}+h d x^{-} d x^{-}+d x_{m}^{2}
$$

where $h=q / r^{7}$. Using the spherical coordinate on $S^{8}$, the metric reads

$$
g_{++}=0, g_{+-}=\frac{1}{2}, g_{--}=h, g_{r r}=1, g_{i j}=r^{2} k_{i j}
$$

where $k_{i j}$ is the metric of the unit sphere $S^{8}$ and $x^{i}(i, j=1, \ldots 8)$ are the (angular) coordinate along $S^{8}$.

Non-vanishing components of the Christoffel symbols are

$$
\Gamma_{--}^{r}=-\frac{1}{2} h^{\prime}, \Gamma_{-r}^{+}=h^{\prime}, \Gamma_{j r}^{i}=\frac{1}{r} \delta_{j}^{i}, \Gamma_{i j}^{r}=-\frac{1}{r} g_{i j}, \Gamma_{j k}^{i}=\Gamma_{j k}^{i}\left(S^{8}\right)
$$

where $\Gamma_{j k}^{i}{ }^{\left(S^{8}\right)}$ means the Christoffel symbol on the unit sphere $S^{8}$ and $h^{\prime}=\partial_{r} h$. Nonvanishing components of the curvature tensors are

$$
R_{-j-}^{i}=-\frac{h^{\prime}}{2 r} \delta_{k}^{i}=\frac{7}{2} \frac{q}{r^{9}} \delta_{k}^{i}, \quad R_{-r-}^{r}=-\frac{1}{2} h^{\prime \prime}=-28 \frac{q}{r^{9}} .
$$

With the choice of the vielbein

$$
e_{+}^{\hat{0}}=\frac{1}{2 \sqrt{h}}, \quad e^{\hat{11}}+=\frac{1}{2 \sqrt{h}}, \quad e_{-}^{\hat{0}}=0, \quad e^{\hat{11}}=\sqrt{h},
$$

non-vanishing components of the spin connections are

$$
\omega_{-\hat{0} \hat{r}}=-\frac{h^{\prime}}{2 \sqrt{h}}, \omega_{-\hat{1} \hat{1} \hat{r}}=\frac{h^{\prime}}{2 \sqrt{h}}, \omega_{r \hat{0} \hat{1} 1}=-\frac{h^{\prime}}{2 h}, \omega_{i \hat{r} \hat{\jmath}}=-\frac{1}{r} e_{\hat{\jmath} i} e_{\hat{r} r}, \omega_{i \hat{\jmath} \hat{k}}=\omega_{i \hat{\jmath} \hat{k}}\left(S^{8}\right)
$$

where $\omega_{i \hat{\jmath} \hat{k}}\left(S^{8}\right)$ means the spin connection on the unit sphere $S^{8}$. 
We define the covariant derivative which is made from the connections on $S^{8}, \tilde{D}_{i}$. The covariant derivative $D_{i}$ has extra contributions from $\Gamma^{r}{ }_{i j}, \Gamma_{r j}^{i}, \omega_{i r \hat{\jmath}}$. For example, the covariant derivative of a spinor $\epsilon$ is

$$
\begin{aligned}
D_{i} \epsilon & =\partial_{i} \epsilon+\frac{1}{4} \omega_{i \hat{M} \hat{N}} \Gamma^{\hat{M} \hat{N}} \epsilon=\tilde{D}_{i} \epsilon+\frac{1}{2} \omega_{i \hat{r} \hat{j}} \hat{\Gamma}^{\hat{j} \hat{j}} \epsilon \\
& =\tilde{D}_{i} \epsilon-\frac{1}{2}\left(\sigma^{3} \otimes \gamma^{9} \gamma_{i}\right) \epsilon
\end{aligned}
$$

using the representation of the $\Gamma$ matrix (3.8).

\section{B. Spectrum of the $\Xi^{k=0,+}$ mode}

In this appendix, we describe the details of the analysis of the spectrum of the $\Xi^{k=0,+}$ mode and show that there is one physical degree of freedom which is solved by the Bessel equation with order $\nu=21 / 5$. We have $2 \times 4$ components of equations (3.2) $\sim(3.5)$ for 8 variables $\left(\hat{\Psi}_{+}^{(1)}, \hat{\Psi}_{+}^{(2)}, \hat{\Psi}_{-}^{(1)}, \hat{\Psi}_{-}^{(2)}, \hat{\Psi}_{r}^{(1)}, \hat{\Psi}_{r}^{(2)}, \hat{\Psi}^{(1)}\right.$ and $\hat{\Psi}^{(2)}$, where the superscripts (1) and (2) denote upper and lower component of the two-component spinors, respectively).

There is a local supersymmetry $\delta \hat{\Psi}_{M}=D_{M} \epsilon$ under which the fields transform as

$$
\begin{aligned}
& \delta \hat{\Psi}_{+}=\partial_{+} \hat{\epsilon}, \quad \delta \hat{\Psi}_{-}=\partial_{-} \hat{\epsilon}+\frac{h^{\prime}}{4 \sqrt{h}}\left(-\sigma^{2}+i \sigma^{1}\right) \hat{\epsilon} \\
& \delta \hat{\Psi}_{r}=\partial_{r} \hat{\epsilon}+\frac{h^{\prime}}{4 h} \sigma^{3} \hat{\epsilon}, \quad \delta \hat{\Psi}=\frac{1}{2}\left(1-\sigma^{3}\right) \hat{\epsilon}
\end{aligned}
$$

The parameter $\hat{\epsilon}$ is defined by $\epsilon=\hat{\epsilon}\left(\gamma^{9}\right)^{-1 / 2} \eta$ where $\eta$ is the Killing spinor on $S^{8}$. We use half of the gauge freedom and set $\hat{\Psi}^{(2)}=0$. Other half will be fixed in the following. The equations of motion in components with the condition $\hat{\Psi}^{(2)}=0$ and $\partial_{-}=0$ are the following. From (3.2),

$$
\begin{aligned}
& -\frac{i}{\sqrt{h}} \partial_{r} \hat{\Psi}_{-}^{(2)}+i\left(\frac{h^{\prime}}{4 h^{3 / 2}}-\frac{8}{\sqrt{h} r}\right) \hat{\Psi}_{-}^{(2)}-\frac{8}{r} \partial_{r} \hat{\Psi}^{(1)}-\left(\frac{56}{r^{2}}+\frac{2 h^{\prime}}{r h}\right) \hat{\Psi}^{(1)}=0 \\
& -\frac{i}{\sqrt{h}} \partial_{r} \hat{\Psi}_{-}^{(1)}-\frac{i h^{\prime}}{4 h^{3 / 2}} \hat{\Psi}_{-}^{(1)}-\left(\frac{8}{r}+\frac{h^{\prime}}{2 h}\right) \hat{\Psi}_{r}^{(2)}=0
\end{aligned}
$$

From (3.3),

$$
\begin{aligned}
& 2 \partial_{+} \hat{\Psi}_{r}^{(1)}-2 \partial_{r} \hat{\Psi}_{+}^{(1)}-\frac{h^{\prime}}{2 h} \hat{\Psi}_{+}^{(1)}+\frac{16 i}{r \sqrt{h}} \hat{\Psi}_{r}^{(2)}-\frac{16}{r} \partial_{+} \hat{\Psi}^{(1)}=0 \\
& 2 \partial_{+} \hat{\Psi}_{r}^{(2)}-2 \partial_{r} \hat{\Psi}_{+}^{(2)}+\left(-\frac{16}{r}+\frac{h^{\prime}}{2 h}\right) \hat{\Psi}_{+}^{(2)}=0
\end{aligned}
$$


From (3.5),

$$
\begin{aligned}
& -2 \partial_{+} \hat{\Psi}_{-}^{(1)}+i \sqrt{h}\left(\frac{16}{r}+\frac{h^{\prime}}{h}\right) \hat{\Psi}_{+}^{(2)}-\frac{16 i}{\sqrt{h} r} \hat{\Psi}_{-}^{(2)}-\frac{56}{r^{2}} \hat{\Psi}^{(1)}=0 \\
& 2 \partial_{+} \hat{\Psi}_{-}^{(2)}-\frac{16 i \sqrt{h}}{r} \partial_{+} \hat{\Psi}^{(1)}=0
\end{aligned}
$$

From (3.6),

$$
\begin{aligned}
& -\frac{2 i}{r \sqrt{h}} \hat{\Psi}_{-}^{(2)}+\frac{2 i \sqrt{h}}{r} \hat{\Psi}_{+}^{(2)}-\frac{1}{r} \partial_{r} \hat{\Psi}^{(1)}-\left(\frac{14}{r^{2}}+\frac{h^{\prime}}{4 r h}\right) \hat{\Psi}^{(1)}=0 \\
& -\frac{1}{r} \hat{\Psi}_{r}^{(2)}-\frac{2 i \sqrt{h}}{r} \partial_{+} \hat{\Psi}^{(1)}=0
\end{aligned}
$$

To solve this set of equations, first note that (B.7) can be written as

$$
\partial_{+}\left(\hat{\Psi}_{-}^{(2)}-\frac{8 i \sqrt{h}}{r} \hat{\Psi}^{(1)}\right)=0
$$

and $(\overline{B .2})$ can be written as

$$
\partial_{r}\left\{r^{8} h^{-1 / 4}\left(\hat{\Psi}_{-}^{(2)}-\frac{8 i \sqrt{h}}{r} \hat{\Psi}^{(1)}\right)\right\}=0,
$$

thus, we can set

$$
\hat{\Psi}_{-}^{(2)}=\frac{8 i \sqrt{h}}{r} \hat{\Psi}^{(1)} .
$$

From $(\mathbb{B} .9)$, we find

$$
\hat{\Psi}_{r}^{(2)}=-2 i \sqrt{h} \partial_{+} \hat{\Psi}^{(1)} .
$$

From (B.8) and (B.10),

$$
\hat{\Psi}_{+}^{(2)}=-\frac{r}{16 h}\left\{\partial_{r} \hat{\Psi}_{-}^{(2)}-\left(\frac{1}{r}+\frac{h^{\prime}}{4 h}\right) \hat{\Psi}_{-}^{(2)}\right\}
$$

Using $(\overline{B .10}) \sim(\overline{B .12})$ to eliminate $\hat{\Psi}^{(1)}, \hat{\Psi}_{r}^{(2)}$ and $\hat{\Psi}_{+}^{(2)}$ (and using $\left.h^{\prime}=-7 h / r\right),($ B.3) can be written as

$$
i \partial_{r}\left(h^{1 / 4} \hat{\Psi}_{-}^{(1)}\right)=\frac{9}{8} h^{3 / 4} \partial_{+} \hat{\Psi}_{-}^{(2)}
$$

and (B.6) can be written as

$$
\partial_{+} \hat{\Psi}_{-}^{(1)}=-\frac{9 i}{32 \sqrt{h}} \partial_{r} \hat{\Psi}_{-}^{(2)}-\frac{603 i}{128 r \sqrt{h}} \hat{\Psi}_{-}^{(2)}
$$


This set of 'Dirac equation' is solved by $\hat{\Psi}_{-}^{(1)}$ which satisfy

$$
\left[-\partial_{t}^{2}+\partial_{z}+\frac{1}{z} \partial_{z}-\left(\frac{21}{5}\right)^{2} \frac{1}{z^{2}}\right] z^{-7 / 2} \hat{\Psi}_{-}^{(1)}=0
$$

where we have reinterpreted $x^{+} \rightarrow 2 t$ and changed the radial variables $z=2 q^{1 / 2} r^{-5 / 2} / 5$.

We have found one physical degree of freedom $\hat{\Psi}_{-}^{(1)}$ and we have seen that the variables $\hat{\Psi}^{(1)}, \hat{\Psi}_{r}^{(2)}, \hat{\Psi}_{+}^{(2)}$ and $\hat{\Psi}_{-}^{(2)}$ are determined using the equations $(\mathrm{B} .10) \sim(\mathrm{B} .13)$. Remaining 2 variables, $\hat{\Psi}_{+}^{(1)}$ and $\hat{\Psi}_{r}^{(1)}$ are determined by $(\mathbb{B . 4})$ and using the remaining gauge freedom (to set e.g. $\hat{\Psi}_{+}^{(1)}=0$ ). We can check that the set of solutions satisfy the equation (B.5) which we have not used for the derivation, which shows that the system of the equations is consistent.

\section{References}

[1] T. Banks, W. Fischler, S. H. Shenker and L. Susskind, "M-Theory as a Matrix Model: A Conjecture", Phys. Rev. D55 (1997) 5112, hep-th/9610043.

[2] L. Susskind, "Another Conjecture about M(atrix) Theory", hep-th/9704080.

[3] N. Seiberg, "Why is the Matrix Model Correct?", Phys. Rev. Lett. 79 (1997) 3577, hep-th/9710009.

[4] A. Sen, "D0 Branes on T ${ }^{n}$ and Matrix Theory", Adv. Theor. Math. Phys. 2 (1998) 51 , hep-th/9709220.

[5] K. Becker and M. Becker, "A Two-Loop Test of M(atrix) Theory", Nucl. Phys. B506 (1997) 48, hep-th/9705091.

[6] K. Becker, M. Becker, J. Polchinski and A. Tseytlin, "Higher Order Graviton Scattering in M(atrix) Theory", Phys. Rev. D56 (1997) 3174, hep-th/9706072.

[7] Y. Okawa and T. Yoneya, "Multi-Body Interactions of D-Particles in Supergravity and Matrix Theory", Nucl. Phys. B538 (1998) 67, hep-th/9806108; "Equations of Motion and Galilei Invariance in D-Particle Dynamics", Nucl. Phys. B541 (1999) 163, hep-th/9808188. 
[8] J. Maldacena, "The Large $N$ Limit of Superconformal Field Theories and Supergravity", Adv. Theor. Math. Phys. 2 (1998) 231, hep-th/9711200.

[9] N. Itzhaki, J. Maldacena, J. Sonnenschein and S. Yankielowicz, "Supergravity and The Large N Limit of Theories With Sixteen Supercharges", Phys. Rev. D58 (1998) 046004, hep-th/9802042.

[10] A. Jevicki and T. Yoneya, "Space-Time Uncertainty Principle and Conformal Symmetry in D-Particle Dynamics", Nucl. Phys. B535 (1998) 335, hep-th/9805069.

[11] Y. Sekino and T. Yoneya, "Generalized AdS-CFT Correspondence for Matrix Theory in the Large N limit", Nucl. Phys. B570 (2000) 174, hep-th/9907029.

[12] S. S. Gubser, I. R. Klebanov and A. M. Polyakov, "Gauge Theory Correlators from Noncritical String Theory", Phys. Lett. B428 (1998) 105, hep-th/9802109.

[13] E. Witten, "Anti de Sitter Space and Holography", Adv. Theor. Math. Phys. 2 (1998) 505, hep-th/9802150.

[14] T. Banks, N. Seiberg and S. Shenker, "Branes from Matrices", Nucl. Phys. B490 (1997) 91, hep-th/9612157.

[15] S. Hyun, Y. Kiem and H. Shin, "Infinite Lorentz boost along the M-theory circle and non-asymptotically flat solutions in supergravities", Phys. Rev. D57 (1998) 4856, hep-th/9712021.

[16] S. Hyun, "The Background Geometry of DLCQ Supergravity", Phys. Lett. B441 (1998) 116, hep-th/9802026.

[17] S. Hyun and Y. Kiem, "Background geometry of DLCQ M theory on a p-torus and holography", Phys. Rev. D59 (1999) 026003, hep-th/9805136.

[18] P. van Niewenhuizen, "The complete mass spectrum of $d=11$ supergravity compactified on $S_{4}$ and a general mass formula for arbitrary cosets $M_{4}$ ", Class. Quantum Grav. 2 (1985) 1. 
[19] M. Henningson and K. Sfetsos, "Spinors and the AdS/CFT Correspondence", Phys. Lett. B431 (1998) 63, hep-th/9803251.

[20] M. Henneaux, "Boundary terms in the AdS/CFT correspondence for spinor fields", hep-th/9902137.

[21] W. Taylor and M. Van Raamsdonk, "Supergravity currents and linearized interactions for Matrix Theory configurations with fermionic backgrounds", JHEP 9904 (1999) 013, hep-th/9812239.

[22] A. Dasgupta, H. Nicolai and J. Plefka, "Vertex Operators for the Supermembrane", JHEP 0005 (2000) 007, hep-th/0003280.

[23] W. Taylor and M. Van Raamsdonk, "Multiple D0-branes in Weakly Curved Backgrounds", Nucl.Phys. B558 (1999) 63-95, hep-th/9904095.

[24] W. Taylor and M. Van Raamsdonk, "Multiple Dp-branes in Weak Background Fields", Nucl.Phys. B573 (2000) 703-734, hep-th/9910052.

[25] I. Klebanov, W. Taylor and M. Van Raamsdonk, "Absorption of dilaton partial waves by D3-branes", Nucl.Phys. B560 (1999) 207-229, hep-th/9905174.

[26] J. Polchinski, "M-Theory and the Light Cone", Prog. Theor. Phys. Suppl. 134 (1999) 158, hep-th/9903165.

[27] T. Yoneya, "Generalized Conformal Symmetry and Oblique AdS/CFT Correspondence for Matrix Theory", hep-th/9908153.

[28] J. de Boer, E. Verlinde and H. Verlinde, "On the Holographic Renormalization Group", JHEP 0008 (2000) 003, hep-th/9912012.

[29] D. Kabat and G. Lifschytz, "Approximations for Strongly-Coupled Supersymmetric Quantum Mechanics", Nucl.Phys. B571 (2000) 419, hep-th/9910001.

[30] D. Kabat, G. Lifschytz and D. A. Lowe, "Black Hole Thermodynamics from Calculations in Strongly-Coupled Gauge Theory", hep-th/0007051. 
[31] R. Dijkgraaf, E. Verlinde and H. Verlinde, "Matrix String Theory", Nucl.Phys. B500 (1997) 43, hep-th/9703030. 